\title{
Knowledge, perception and attitude of under-graduate and post- graduate medical students about antimicrobial use, resistance and stewardship at a tertiary care teaching hospital in rural Telangana, India
}

\author{
Jayaram Chundru ${ }^{1}$, Shailendra D. ${ }^{1 *}$, Rahul R. Tirumalareddy ${ }^{2}$, \\ Harsha Kumari ${ }^{2}$, Vishal P. Kovilakonda ${ }^{2}$
}

\begin{abstract}
${ }^{1}$ Department of Pharmacology, ${ }^{2}$ Intern, MediCiti Institute of Medical Sciences, Hyderabad, Telangana, India
\end{abstract}

Received: 09 January 2019 Accepted: 16 January 2019

\section{*Correspondence to: \\ Dr. Shailendra D., \\ Email: shailendra962@ gmail.com}

Copyright: (c) the author(s), publisher and licensee Medip Academy. This is an openaccess article distributed under the terms of the Creative Commons Attribution NonCommercial License, which permits unrestricted noncommercial use, distribution, and reproduction in any medium, provided the original work is properly cited.

\begin{abstract}
Background: Inappropriate antimicrobial use contributes to antimicrobial resistance. Assessing knowledge, perception and attitude regarding antimicrobial use serves as a prelude to design and implement educational modules to promote rational antimicrobial use.

Methods: An online questionnaire based cross-sectional study involving 338 medical students; under-graduates, interns and post-graduates was conducted in a medical college in south India. Chi-square test or Fisher exact test were used to determine association between variables.

Results: Fifty percent of the participants (170/338) responded to the questionnaire. The responses were similar across undergraduates, interns and post-graduates. Majority of the responders had good knowledge regarding use of antimicrobials. Most of the participants $(92.35 \%)$ expressed that a broadspectrum antimicrobial should be started to treat a serious infection while awaiting culture and sensitivity reports and $88.82 \%$ did not prefer to use antimicrobial agents for common cold. Similarly, most of the respondents $(93.56 \%)$ were aware of the fact that antimicrobial resistance is a global problem. However, many of them $(74.71 \%)$ were not aware of antimicrobial stewardship programme in their college.

Conclusions: This study revealed that most of the students had good knowledge, fair perception and positive attitude regarding the use of, and resistance to antimicrobial agents but not about stewardship programmes. Proactive measures are required to sensitise medical students on antimicrobial stewardship programmes.
\end{abstract}

Keywords: Antimicrobial stewardship, Attitude, Knowledge, Medical students, Perception, Resistance

\section{INTRODUCTION}

Resistance to antimicrobial agents presents a pernicious menace to the entire globe. The problem of resistance to antimicrobial agents is progressing rapidly. Globally, irrational use of antimicrobials is estimated to be $50 \%$. Awareness on seriousness of the resistance is initial measure towards confining its further progress. Prescribers play a prominent role in the prevention of resistance; by - rational prescribing, providing health education and also by promoting patient awareness pertaining to safe medication practices in the community with respect to antimicrobials. ${ }^{2}$ Key to successful antimicrobial therapy lies with correct diagnosis followed by right drug in the right dose by right route of administration at right frequency for the right duration. ${ }^{3}$ This rightness can possibly lessen or probably check the evolution of resistant 
strains of organisms as conceptualized by antimicrobial stewardship programmes.

Antimicrobial stewardship involves optimal, cautious use of antimicrobial agents for patients across the sequence of care i.e., acute, outpatient settings, inpatient, and longterm care. Emergence of multiple drug resistant organisms in the current health scenario across the world is alarming and the possibility of checking such acquired form of resistance is optimally done by complying with stringent antimicrobial stewardship programme at the level of every stake holder i.e., prescriber, hospitals, community and lastly, national guidelines with relevance to locally prevalent resistance patterns. ${ }^{4}$ It is important that the prescribers become cognizant of the local resistance patterns and all the effective antimicrobial options available for the successful treatment of such infections. ${ }^{5}$ The future generations of the physicians may have to face even more demanding scenarios, to prevent and deal with resistance to antimicrobial agents. A student starts practicing only after final MBBS during the internship programme. Rational prescription writing skills are to be acquired at the earliest. Post-graduate students start prescribing drugs usually in the first year. Their mind-set toward good prescribing and rational use of antimicrobials is of prime importance as they are the doctors of the next generation. It thus seems reasonable to conclude that our attempts to nurture rational antimicrobial use should also commence during this period. ${ }^{6}$

With all the above mentioned facts, we intended to assess the current level of understanding of under-graduate and post-graduate medical students with respect to rational use of antimicrobial agents, to effectively treat infections with least possible risk of escalating resistance to antimicrobial agents. This information will help to devise educational modules in existing curriculum to improve rational, judicious and responsible prescriptions of antimicrobial agents that are effective in preventing antimicrobial resistance. Hence, this study was initiated with the objective to assess knowledge, perception and attitude of under-graduate and post-graduate medical students of a tertiary care teaching hospital regarding rational use of antimicrobials, antimicrobial resistance and stewardship.

\section{METHODS}

This was an online questionnaire based cross-sectional study. A total of 338 medical students including undergraduates, interns and post-graduate students from tertiary care teaching medical institute in rural Telangana participated in the study, after getting approval from the institutional ethics committee. Total duration of the study was for 2 weeks, carried out during the second and third weeks of October 2018. A written informed consent was taken from all those who were willing to participate, after briefly describing about the objectives of the study. All such voluntary participants were provided with online access link, by email, to the study questionnaire which was administered via Google forms. Study questionnaire was well-structured with 29 questions, developed by us and validated by the multidisciplinary team of senior faculty members from the Departments of Pharmacology, Microbiology and Community Medicine.

Each student was given a total time of 15 minutes to answer the questionnaire by choosing the right options which he/she felt to be the most befitting. The students' knowledge aspect (Table 1) was assessed by using a set of 10 questions - seven questions of these are true/false/don't know type, two questions were of yes/no type and one question was of yes/no/may be type. The students' perception aspect (Table 2) was assessed by using a set of nine questions - six questions were of yes/no/may be type, two questions were of agree/disagree/don't know type and one question was of true/false/don't know type. The students' attitude aspect (Table 3) was assessed by using a set of ten questions - eight questions were of yes/no/may be type, one question each of A/B/anyone/both type and yes/no type.

\section{Statistical analysis}

An overall score was assessed by calculating the total percentage of correct answers for the knowledge, perception and attitude aspects. Upon completion of the study, all the data were entered in spreadsheet and statistical analysis was done by applying descriptive statistics to generate frequencies, proportions and percentages. Chi-square test or Fisher exact test were used to determine association between variables, as appropriate. MedCalc statistical software was used to perform all statistical analyses.

\section{RESULTS}

A total of 338 students consented for the study, of which only 170 completed the survey. All the participants answered all the questions. Out of 170 participants, 26 were of final MBBS-I, 39 were of final MBBS-II, 82 were interns and 23 were post-graduate (PG) students.

Amongst knowledge based questions, maximum correct responses were for the question no. 6 by all the subgroups viz., Final MBBS-I students (100\%), final MBBS-II students $(100 \%)$, interns $(98.78 \%)$, and post-graduate (PG) student (100\%). Maximum correct responses were also for question no. 10 by final MBBS-II students (100\%), and for question no. 4 and 10 by PG students (100\%). While, least correct responses were for question no. 2 by final MBBS-I students $(46.15 \%)$ and for question no. 1 and question no. 2 by final MBBS-II students $(53.85 \%)$, for question no. 7 by Interns (45.12\%) and PG students $(34.78 \%)$. There is no statistically significant difference between groups for knowledge based questions except for question no. 7 ( $\mathrm{p}=$ $0.046)$ and $8(\mathrm{p}=0.010)$. All the data regarding participants' knowledge in relation to rational antimicrobial use, resistance and antimicrobial stewardship are depicted in Table 1. 
Table 1: Knowledge about antimicrobial use, resistance and stewardship.

\begin{tabular}{|c|c|c|c|c|c|c|c|}
\hline Questions & & $\begin{array}{l}\text { MBBS-I } \\
\text { n (\%) }\end{array}$ & $\begin{array}{l}\text { MBBS-II } \\
\text { n }(\%)\end{array}$ & $\begin{array}{l}\text { Intern } \\
\mathrm{n}(\%)\end{array}$ & $\begin{array}{l}\text { PG student } \\
\text { n (\%) }\end{array}$ & Total n (\%) & p value \\
\hline \multirow{3}{*}{$\begin{array}{l}\text { Clavulanic acid is an } \\
\text { antimicrobial agent }\end{array}$} & Yes & $14(53.85)$ & $21(53.85)$ & $47(57.32)$ & $17(73.91)$ & $99(58.24)$ & \multirow{3}{*}{$0.4804 *$} \\
\hline & No & $10(38.46)$ & $14(35.90)$ & $32(39.02)$ & $6(26.09)$ & $62(36.47)$ & \\
\hline & May be & $2(7.69)$ & $4(10.26)$ & $3(3.66)$ & $0(0.00)$ & $9(5.29)$ & \\
\hline \multirow{3}{*}{$\begin{array}{l}\text { Rifampicin can be } \\
\text { used as monotherapy } \\
\text { for certain indications }\end{array}$} & True & $12(46.15)$ & $21(53.85)$ & 55 (67.07) & $16(69.57)$ & $104(61.18)$ & \multirow{3}{*}{$0.2389 *$} \\
\hline & False & $11(42.31)$ & $10(25.64)$ & $15(18.29)$ & $5(21.74)$ & $41(24.12)$ & \\
\hline & $\begin{array}{l}\text { Don't } \\
\text { know }\end{array}$ & $3(11.54)$ & $8(20.51)$ & $12(14.63)$ & $2(8.70)$ & 25 (14.71) & \\
\hline \multirow{3}{*}{$\begin{array}{l}\text { Aminoglycosides are } \\
\text { not effective in } \\
\text { urinary tract } \\
\text { infections }\end{array}$} & True & $12(46.15)$ & $5(12.82)$ & $22(26.83)$ & $4(17.39)$ & $43(25.29)$ & \multirow{3}{*}{$0.0726^{*}$} \\
\hline & False & $14(53.85)$ & $33(84.62)$ & $57(69.51)$ & $18(78.26)$ & $122(71.76)$ & \\
\hline & $\begin{array}{l}\text { Don't } \\
\text { know }\end{array}$ & $0(0.00)$ & $1(2.56)$ & $3(3.66)$ & $1(4.35)$ & $5(2.94)$ & \\
\hline \multirow{3}{*}{$\begin{array}{l}\text { Antimicrobial } \\
\text { stewardship aims at } \\
\text { improving patient } \\
\text { outcomes and } \\
\text { reducing risk of } \\
\text { antimicrobial } \\
\text { resistance }\end{array}$} & True & $22(84.62)$ & $28(71.79)$ & $65(79.27)$ & $23(100)$ & $138(81.18)$ & \multirow[b]{3}{*}{$0.1415^{*}$} \\
\hline & False & $1(3.85)$ & $3(7.69)$ & $3(3.66)$ & $0(0.00)$ & $7(4.12)$ & \\
\hline & $\begin{array}{l}\text { Don't } \\
\text { know }\end{array}$ & $3(11.54)$ & $8(20.51)$ & $14(17.07)$ & $0(0.00)$ & $25(14.71)$ & \\
\hline \multirow{3}{*}{$\begin{array}{l}\text { Antibiotics are useful } \\
\text { in treating viral } \\
\text { infections }\end{array}$} & True & $10(38.46)$ & $8(20.51)$ & $23(28.05)$ & $2(8.70)$ & $43(25.29)$ & \multirow{3}{*}{$0.0510^{*}$} \\
\hline & False & $14(53.85)$ & $29(74.36)$ & $58(70.73)$ & $21(91.30)$ & $122(71.76)$ & \\
\hline & $\begin{array}{l}\text { Don't } \\
\text { know }\end{array}$ & $2(7.69)$ & $2(5.13)$ & $1(1.22)$ & $0(0.00)$ & $5(2.94)$ & \\
\hline \multirow{2}{*}{$\begin{array}{l}\text { Have you ever heard } \\
\text { of antimicrobial } \\
\text { resistance }\end{array}$} & Yes & $26(100)$ & $39(100)$ & $81(98.78)$ & $23(100)$ & $169(99.41)$ & \multirow[b]{2}{*}{$1 *$} \\
\hline & No & $0(0.00)$ & $0(0.00)$ & $1(1.22)$ & $0(0.00)$ & $1(0.59)$ & \\
\hline \multirow{2}{*}{$\begin{array}{l}\text { Have you ever heard } \\
\text { of antimicrobial } \\
\text { stewardship }\end{array}$} & Yes & $8(30.77)$ & $16(41.03)$ & $45(54.88)$ & $15(65.22)$ & $84(49.41)$ & \multirow[b]{2}{*}{$0.0462^{\#}$} \\
\hline & No & $18(69.23)$ & $23(58.97)$ & $37(45.12)$ & $8(34.78)$ & 86 (50.59) & \\
\hline \multirow{3}{*}{$\begin{array}{l}\text { While awaiting } \\
\text { culture and sensitivity } \\
\text { reports of a serious } \\
\text { infection it is } \\
\text { advisable to start a } \\
\text { broad spectrum } \\
\text { antimicrobial agent }\end{array}$} & True & $20(76.92)$ & 38 (97.44) & $79(96.34)$ & $20(86.96)$ & $157(92.35)$ & \multirow[b]{3}{*}{$0.0101 *$} \\
\hline & False & $5(19.23)$ & $1(2.56)$ & $2(2.44)$ & $3(13.04)$ & $11(6.47)$ & \\
\hline & $\begin{array}{l}\text { Don't } \\
\text { know }\end{array}$ & $1(3.85)$ & $0(0.00)$ & $1(1.22)$ & $0(0.00)$ & $2(1.18)$ & \\
\hline \multirow{3}{*}{$\begin{array}{l}\text { Antimicrobials can } \\
\text { cause secondary } \\
\text { infections by killing } \\
\text { good bacteria in the } \\
\text { body }\end{array}$} & True & $25(96.15)$ & $33(84.62)$ & $62(75.61)$ & $20(86.96)$ & $140(82.35)$ & \multirow[b]{3}{*}{$0.1009 *$} \\
\hline & False & $1(3.85)$ & $2(5.13)$ & $15(18.29)$ & $3(13.04)$ & $21(12.35)$ & \\
\hline & $\begin{array}{l}\text { Don't } \\
\text { know }\end{array}$ & $0(0.00)$ & $4(10.26)$ & $5(6.10)$ & $0(0.00)$ & $9(5.29)$ & \\
\hline \multirow{3}{*}{$\begin{array}{l}\text { Antimicrobials can } \\
\text { cause allergic } \\
\text { reactions }\end{array}$} & True & $25(96.15)$ & $39(100)$ & $79(96.34)$ & $23(100)$ & $166(97.65)$ & \multirow{3}{*}{$0.6349 *$} \\
\hline & False & $1(3.85)$ & $0(0.00)$ & $3(3.66)$ & $0(0.00)$ & $4(2.35)$ & \\
\hline & $\begin{array}{l}\text { Don't } \\
\text { know }\end{array}$ & $0(0.00)$ & $0(0.00)$ & $0(0.00)$ & $0(0.00)$ & $0(0.00)$ & \\
\hline
\end{tabular}

Values expressed as n (\%); * - Fisher's exact test; \# - Chi square test; $\mathrm{p} \leq 0.05$ is considered as statistically significant.

Regarding perception based questions, maximum correct response were for question no. 6 by final MBBS-I (100\%); for question no. 4 and 6 by final MBBS-II students (92.31\%); for question no. 3 and 6 by interns $(93.90 \%)$ and for question no. 3, 4 and 6 by PG students (100\%). While, least correct responses were for question no. 8 by final
MBBS-I students (42.31\%), Interns (41.46\%), and PG students $(52.17 \%)$ and for question no. 7 by final MBBSII students $(46.15 \%)$. There was no statistically significant difference between groups for perception based questions. All the data regarding participants' perception towards rational antimicrobial use, resistance and antimicrobial stewardship are depicted in Table 2. 
Table 2: Perception of antimicrobial use, resistance and stewardship.

\begin{tabular}{|c|c|c|c|c|c|c|c|}
\hline Questions & & $\begin{array}{l}\text { MBBS-I } \\
\text { n }(\%)\end{array}$ & $\begin{array}{l}\text { MBBS-II } \\
\text { n }(\%)\end{array}$ & $\begin{array}{l}\text { Interns } \\
\text { n }(\%)\end{array}$ & $\begin{array}{l}\text { PG } \\
\text { n }(\%)\end{array}$ & Total n (\%) & $p$ value \\
\hline \multirow{3}{*}{$\begin{array}{l}\text { All antimicrobials are } \\
\text { effective in-vitro are } \\
\text { also effective in-vivo }\end{array}$} & True & $8(30.77)$ & $4(10.26)$ & $11(13.41)$ & $2(8.70)$ & $25(14.71)$ & \multirow{3}{*}{$0.3870 *$} \\
\hline & False & $16(61.54)$ & $32(82.05)$ & $65(79.27)$ & $19(82.61)$ & $132(77.65)$ & \\
\hline & $\begin{array}{l}\text { Don't } \\
\text { know }\end{array}$ & $2(7.69)$ & $3(7.69)$ & $6(7.32)$ & $2(8.70)$ & $13(7.65)$ & \\
\hline \multirow{3}{*}{$\begin{array}{l}\text { Do you think pain and } \\
\text { inflammation without } \\
\text { any possibility of } \\
\text { infection are indications } \\
\text { for antimicrobial } \\
\text { therapy? }\end{array}$} & Yes & $3(11.54)$ & $7(17.95)$ & $10(12.20)$ & $1(4.35)$ & $21(12.35)$ & \multirow[b]{3}{*}{$0.5087^{*}$} \\
\hline & No & $20(76.92)$ & $29(74.36)$ & 65 (79.27) & $22(95.65)$ & $136(80.00)$ & \\
\hline & May be & $3(11.54)$ & $3(7.69)$ & $7(8.54)$ & $0(0.00)$ & $13(7.65)$ & \\
\hline \multirow{3}{*}{$\begin{array}{l}\text { Do you think } \\
\text { antimicrobial resistance } \\
\text { is a problem world- } \\
\text { wide? }\end{array}$} & Yes & $24(92.31)$ & $35(89.74)$ & 77 (93.90) & $23(100)$ & $159(93.56)$ & \multirow{3}{*}{$0.7400 *$} \\
\hline & No & $0(0.00)$ & $1(2.56)$ & $2(2.44)$ & $0(0.00)$ & $3(1.76)$ & \\
\hline & May be & $2(7.69)$ & $3(7.69)$ & $3(3.66)$ & $0(0.00)$ & $8(4.71)$ & \\
\hline \multirow{3}{*}{$\begin{array}{l}\text { Antimicrobial } \\
\text { resistance leads to } \\
\text { increased morbidity and } \\
\text { health care costs }\end{array}$} & Yes & $24(92.31)$ & $36(92.31)$ & 7 & $23(1$ & $.53)$ & \multirow{3}{*}{$0.8255^{*}$} \\
\hline & No & $0(0.00)$ & $0(0.00)$ & $1(1.22)$ & $0(0.00)$ & $1(0.59)$ & \\
\hline & May be & $2(7.69)$ & $3(7.69)$ & $5(6.10)$ & $0(0.00)$ & $10(5.88)$ & \\
\hline \multirow{3}{*}{$\begin{array}{l}\text { Chemoprophylaxis may } \\
\text { increase the risk of } \\
\text { antimicrobial resistance }\end{array}$} & Yes & $20(76.92)$ & $25(64.10)$ & $51(62.20)$ & $18(78.26)$ & $114(67.06)$ & \multirow{3}{*}{$0.1502 *$} \\
\hline & No & $3(11.54)$ & $5(12.82)$ & $21(25.61)$ & $1(4.35)$ & 65) & \\
\hline & May be & $3(11.52)$ & $9(23.08)$ & $10(12.20)$ & $4(17.39)$ & 26 & \\
\hline \multirow{3}{*}{$\begin{array}{l}\text { Do you think misuse of } \\
\text { antimicrobials can lead } \\
\text { to antimicrobial } \\
\text { resistance? }\end{array}$} & Yes & $26(100.00$ & $36(92.31)$ & $77(93.90)$ & $23(100)$ & $5.29)$ & \multirow{3}{*}{$0.8322 *$} \\
\hline & No & $0(0.00)$ & $1(2.56)$ & $1(1.22)$ & $0(0.00)$ & $2(1.18)$ & \\
\hline & May be & $0(0.00)$ & $2(5.13)$ & $4(4.88)$ & $0(0.00)$ & $6(3.53)$ & \\
\hline \multirow{3}{*}{$\begin{array}{l}\text { Antimicrobial } \\
\text { resistance is more } \\
\text { common with oral } \\
\text { drugs than parenteral } \\
\text { drugs }\end{array}$} & Disagree & $8(30.77)$ & $13(33.33)$ & $17(20.73)$ & $6(26.09)$ & $44(25.88)$ & \multirow{3}{*}{$0.1172^{\#}$} \\
\hline & Agree & $12(46.15)$ & $18(46.15)$ & $44(53.66)$ & $17(73.91)$ & $91(53.53)$ & \\
\hline & $\begin{array}{l}\text { Don't } \\
\text { know }\end{array}$ & $6(23.08)$ & $8(20.51)$ & $21(25.61)$ & $0(0.00)$ & 35 (20.59) & \\
\hline \multirow{3}{*}{$\begin{array}{l}\text { Immuno-compromised } \\
\text { state is a source of drug } \\
\text { resistance }\end{array}$} & Disagree & $10(38.46)$ & $13(33.333)$ & $35(42.68)$ & $8(34.78)$ & $66(38.82)$ & \multirow{3}{*}{$0.9298^{\#}$} \\
\hline & Agree & $11(42.31)$ & $20(51.28)$ & $34(41.46)$ & $12(52.17)$ & $77(45.29)$ & \\
\hline & $\begin{array}{l}\text { Don't } \\
\text { know }\end{array}$ & $5(19.23)$ & $6(15.38)$ & $13(15.85)$ & $3(13.04)$ & $27(15.88)$ & \\
\hline \multirow{3}{*}{$\begin{array}{l}\text { Do you think } \\
\text { antimicrobial } \\
\text { stewardship can control } \\
\text { emergence of resistant } \\
\text { organisms? }\end{array}$} & Yes & $15(57.69)$ & $24(61.54)$ & $52(63.41)$ & $17(73.91)$ & $108(63.53)$ & \multirow[b]{3}{*}{$0.7118^{*}$} \\
\hline & No & $1(3.85)$ & $3(7.69)$ & $2(2.44)$ & $0(0.00)$ & $6(3.53)$ & \\
\hline & May be & $10(38.46)$ & $12(30.77)$ & $28(34.15)$ & $6(26.09)$ & $56(32.94)$ & \\
\hline
\end{tabular}

Values expressed as n (\%); * Fisher's exact test; \# - Chi square test; $\mathrm{p} \leq 0.05$ is considered as statistically significant.

As far as attitude based questions are concerned, maximum correct responses were for the question no. 6 by final MBBS-I students $(88.46 \%)$; question no. 5 by final MBBSII students (89.74\%); question no. 2, 5 and 7 by interns (93.90\%); and question no. 2 and 7 by PG students (100\%). While, least correct responses were for question no. 10 by final MBBS-I students (34.62\%), final MBBS-II students $(12.82 \%)$, and interns $(45.12 \%)$, and for question no. 4 and 9 by PG students (65.22\%). There is no statistically significant difference between groups for attitude based questions except for question no. $7(\mathrm{p}=0.0478)$ and 10
( $<<0.0001)$. All the data regarding participants' attitude towards antimicrobial use, resistance and antimicrobial stewardship are depicted in Table 3.

\section{DISCUSSION}

This study provides an insight into the knowledge, perception and attitude of antimicrobial agents use and resistance among under-graduate students, interns and post-graduate students in this medical college. 
Table 3: Attitude towards antimicrobial use, resistance and stewardship.

\begin{tabular}{|c|c|c|c|c|c|c|c|}
\hline Questions & & $\begin{array}{l}\text { MBBS-I } \\
\text { n }(\%)\end{array}$ & $\begin{array}{l}\text { MBBS-II } \\
\text { n }(\%)\end{array}$ & $\begin{array}{l}\text { Intern } \\
\text { n (\%) }\end{array}$ & $\begin{array}{l}\text { PG } \\
\text { n }(\%)\end{array}$ & Total n (\%) & p value \\
\hline \multirow{3}{*}{$\begin{array}{l}\text { Do you prefer to use } \\
\text { antibiotics for } \\
\text { common cold }\end{array}$} & Yes & $1(3.85)$ & $2(5.13)$ & $3(3.66)$ & $0(0.00)$ & $6(3.53)$ & \multirow{3}{*}{$0.9105^{*}$} \\
\hline & No & $22(84.62)$ & $35(89.71)$ & $72(87.80)$ & $22(95.65)$ & $151(88.82)$ & \\
\hline & May be & $3(11.54)$ & $2(5.13)$ & $7(8.54)$ & $1(4.35)$ & $13(7.65)$ & \\
\hline \multirow{3}{*}{$\begin{array}{l}\text { Do you prefer to use } \\
\text { antimicrobials for all } \\
\text { types of fever }\end{array}$} & Yes & $2(7.69)$ & $2(5.13)$ & $2(2.44)$ & $0(0.00)$ & $6(3.53)$ & \multirow{3}{*}{$0.3867 *$} \\
\hline & No & $21(80.77)$ & $33(84.62)$ & $74(90.24)$ & $23(100)$ & $151(88.82)$ & \\
\hline & May be & $3(11.54)$ & $4(10.26)$ & $6(7.32)$ & $0(0.00)$ & $13(7.65)$ & \\
\hline \multirow{3}{*}{$\begin{array}{l}\text { Do you stop taking } \\
\text { antimicrobials when } \\
\text { you feel better }\end{array}$} & Yes & $4(15.38)$ & $7(17.95)$ & $8(9.76)$ & $2(8.70)$ & $21(12.35)$ & \multirow{3}{*}{$0.4352 *$} \\
\hline & No & $19(73.08)$ & $31(79.49)$ & $68(82.93)$ & $21(91.30)$ & $139(81.79)$ & \\
\hline & May be & $3(11.54)$ & $1(2.56)$ & $6(7.32)$ & $0(0.00)$ & $10(5.88)$ & \\
\hline \multirow{3}{*}{$\begin{array}{l}\text { Do you recommend } \\
\text { to keep left over } \\
\text { antimicrobials for } \\
\text { future use }\end{array}$} & Yes & $4(15.38)$ & $8(20.51)$ & $18(21.95)$ & $5(21.74)$ & $35(20.59)$ & \multirow{3}{*}{$0.3983^{*}$} \\
\hline & No & $17(65.38)$ & $30(76.92)$ & $52(63.41)$ & $15(65.22)$ & $114(67.09)$ & \\
\hline & May be & $5(19.23)$ & $1(2.56)$ & $12(14.63)$ & $3(13.04)$ & $21(12.35)$ & \\
\hline \multirow{3}{*}{$\begin{array}{l}\text { Do you use } \\
\text { recommend use of } \\
\text { left over } \\
\text { antimicrobials for } \\
\text { cold or fever without } \\
\text { consulting doctor }\end{array}$} & Yes & $2(7.69)$ & $3(7.69)$ & $5(6.10)$ & $1(4.35)$ & $11(6.47)$ & \multirow[b]{3}{*}{$0.9061^{*}$} \\
\hline & No & $22(84.62)$ & 35 (89.74) & $74(90.24)$ & $22(95.65)$ & $153(90.00)$ & \\
\hline & May be & $2(7.69)$ & $1(2.56)$ & $3(3.66)$ & $0(0.00)$ & $6(3.53)$ & \\
\hline \multirow{3}{*}{$\begin{array}{l}\text { Do you buy } \\
\text { encourage buying } \\
\text { antimicrobial without } \\
\text { prescription }\end{array}$} & Yes & $2(7.69)$ & $2(5.13)$ & $4(4.88)$ & $1(4.35)$ & $9(5.29)$ & \multirow{3}{*}{$0.9070^{*}$} \\
\hline & No & $23(88.46)$ & $34(87.18)$ & $72(87.80)$ & $22(95.65)$ & $151(88.82)$ & \\
\hline & May be & $1(3.85)$ & $3(7.69)$ & $6(7.32)$ & $0(0.00)$ & $10(5.88)$ & \\
\hline \multirow{3}{*}{$\begin{array}{l}\text { Do you prefer to start } \\
\text { antimicrobial therapy } \\
\text { without proper } \\
\text { medical examination }\end{array}$} & Yes & $1(3.85)$ & $3(7.69)$ & $4(4.88)$ & $0(0.00)$ & $8(4.71)$ & \multirow{3}{*}{$0.0478^{*}$} \\
\hline & No & $19(73.08)$ & $32(82.05)$ & $74(90.24)$ & $23(100)$ & $149(87.06)$ & \\
\hline & May be & $6(23.08)$ & $4(1.26)$ & $4(4.88)$ & $0(0.00)$ & $14(8.24)$ & \\
\hline \multirow{4}{*}{$\begin{array}{l}\text { when an infective } \\
\text { organism is } \\
\text { susceptible to a broad } \\
\text { spectrum antibiotic A } \\
\text { as well as narrow } \\
\text { spectrum antibiotic B } \\
\text { which drug do you } \\
\text { prefer }\end{array}$} & A & $5(19.23)$ & $11(28.21)$ & $21(25.61)$ & $3(13.04)$ & $40(23.53)$ & \multirow[b]{4}{*}{$0.2969^{\#}$} \\
\hline & $\mathrm{B}$ & $16(61.54)$ & $25(64.10)$ & $51(62.20)$ & $20(86.96)$ & $112(65.88)$ & \\
\hline & Any one & $3(11.54)$ & $3(7.69)$ & $8(9.76)$ & $0(0.00)$ & $14(8.24)$ & \\
\hline & Both & $2(7.69)$ & $0(0.00)$ & $2(2.44)$ & $0(0.00)$ & $4(2.35)$ & \\
\hline \multirow{2}{*}{$\begin{array}{l}\text { Are you aware of } \\
\text { antimicrobial } \\
\text { stewardship program } \\
\text { in your medical } \\
\text { college }\end{array}$} & Yes & $3(11.54)$ & $8(20.51)$ & $24(29.27)$ & $8(34.78)$ & $43(25.29)$ & \multirow[b]{2}{*}{$0.1826^{\#}$} \\
\hline & No & $23(88.46)$ & 31 (79.49) & $58(70.73)$ & $15(65.22)$ & $127(74.71)$ & \\
\hline \multirow{3}{*}{$\begin{array}{l}\text { If an organism shows } \\
\text { resistance to } \\
\text { gentamycin } \\
\text { aminoglycoside and } \\
\text { data on susceptibility } \\
\text { to amikacin } \\
\text { aminoglycoside is } \\
\text { not available do you } \\
\text { still prefer amikacin }\end{array}$} & Yes & $10(38.46)$ & $25(64.10)$ & $24(29.27)$ & $6(26.09)$ & $65(38.24)$ & \multirow[b]{3}{*}{$<0.0001^{\#}$} \\
\hline & No & $9(34.62)$ & $5(12.82)$ & $37(45.12)$ & $17(73.91)$ & $68(40.00)$ & \\
\hline & May be & $7(26.92)$ & $9(23.08)$ & $21(25.61)$ & $0(0.00)$ & 37 (21.76) & \\
\hline
\end{tabular}

It was observed that knowledge regarding antimicrobial agent use and resistance was good but not excellent among the medical students, which is consistent with observation made by Sharma K et al. ${ }^{7}$ 
More than $95 \%$ of the students have heard of antimicrobial resistance, but only $71.76 \%$ of the students think that antibiotics should not be used to treat viral infections; similar results were seen by Kumar BVD et al. ${ }^{8}$ Only $49.41 \%$ of the participants reported to have heard about Antimicrobial Stewardship Programme, this shows the lack of education and implementation strategies regarding various stewardship programs; similar observation was made by Badar $\mathrm{V}$ et al. ${ }^{9}$ Attitude regarding antimicrobial use and resistance was fairly positive. The clinical effectiveness of antimicrobials depends on their correct use i.e. $4 \mathrm{R}$ - right diagnosis, right dose, right drug, right duration. $^{10}$

Majority $(81.79 \%)$ of responders do not stop the course of antimicrobials when they feel better and about $67.09 \%$ of the students do not recommend to keep left over antimicrobials for future use. This is corroborated by study performed by Tayyab K et al, and Yashin AN et al. ${ }^{11,12}$

Large number of participants in present study do not buy or encourage buying antimicrobials without prescription; that is contradictory to observation made by Shubha $\mathrm{R}$ et al. ${ }^{13}$ and Shaik T et al. ${ }^{14}$ Mandated guidelines should be made regarding prescribing antimicrobials and should be enforced strictly at the national level.

Majority (88.82\%) of the students do not prefer antimicrobial agents in common cold and similar finding was observed by Tayyab $\mathrm{K}$ et al, and $\mathrm{Hu} \mathrm{Y}$ et al. ${ }^{11,15}$ Also, majority $(88.82 \%)$ of the students do not prefer to use antimicrobial agents for all types of fever, a finding similar to that observed by Shaik T et al. ${ }^{14}$

About $65 \%$ of the students prefer narrow spectrum antimicrobial over broad spectrum antimicrobial for susceptible infective organism, to decrease the antimicrobial resistance, which is akin to the study performed by Abbo LM et al. ${ }^{16}$ Majority of the responder think that antimicrobial resistance is a worldwide problem and antimicrobial resistance leads to increased morbidity and health care costs. These observations are in concordance with a study done by Kumar BVD et al, Tayyab K et al, and Yashin AN et al. ${ }^{8,11,12}$

Excessive and unnecessary use of antimicrobial agents leads to emergence of antimicrobial resistance in humans and animals. Poor infection control practices, overcrowding or poor hygiene are the risk factors for the spread of bacterial resistant in hospital and the community. Awareness among the students regarding important risk factors and also the mechanism of antibacterial resistance should be instituted. ${ }^{16}$

Over $95 \%$ of the students think that misuse of antimicrobials can lead to antimicrobial resistance. Similar observations were made by Shaik T et al, and Abbo LM et al. ${ }^{14,16}$ About $65 \%$ of the students are of the opinion that antimicrobial stewardship can control emergence of resistant organisms.

\section{CONCLUSION}

This study demonstrates that majority of the medical students have satisfactory knowledge, positive attitude and adequate perception regarding the use of antimicrobial agents and antimicrobial resistance. They were aware of the fact that antimicrobial resistance is a global health issue and that it leads to increase in morbidity and healthcare cost. But these students had poor knowledge, attitude and perception related to antimicrobial stewardship programme. Requisite training should be given to these students in this regard, as antimicrobial stewardship has the potential to decrease the incidence of resistance to antimicrobial agents. Furthermore, there is scope for modification of both under- and post-graduate curriculum and various perspectives of antimicrobial resistance should be integrated in their courses.

Funding: No funding sources Conflict of interest: None declared

Ethical approval: The study was approved by the Institutional Ethics Committee

\section{REFERENCES}

1. Seid MA, Hussen MS. Knowledge and attitude towards antimicrobial resistance among final year undergraduate paramedical students at University of Gondar, Ethiopia. BMC Infectious Dis. 2018 Dec;18(1):312.

2. Afzal Khan AK, Banu G, Reshma KK. Antibiotic resistance and usage-a survey on the knowledge, attitude, perceptions and practices among the medical students of a Southern Indian teaching hospital. J Clin Diag Res. 2013 Aug;7(8):1613-6.

3. M Dryden, Johnson AP, Ashiru-Oredope D, Sharland M. Using antibiotics responsibly: right drug, right time, right dose, right duration. J Antimicrob Chemotherapy. 2011 Nov; 66(11):2441-3.

4. Moody J, Cosgrove SE, Olmsted R, Septimus E, Aureden K, Oriola S, et al. Antimicrobial stewardship: a collaborative partnership between infection preventionists and healthcare epidemiologists. Am J Infect Control. 2012 Mar;40(2):94-5.

5. Alothman A, Algwizani A, Alsulaiman M, Alalwan A, Binsalih S, Bosaeed M. Knowledge and attitude of physicians toward prescribing antibiotics and the risk of resistance in two reference hospitals. Infectious Diseases. 2016 Jan;9:33-8.

6. Dakhale G, Pimpalkhute S, Bajait C, Raghute L. Evaluation of knowledge, attitude and practice of rational use of medicine among interns and resident doctors in a tertiary care teaching hospital. J Young Pharm. 2016; 8(2):114-7.

7. Sharma K, Jain P, Sharma A. Knowledge, attitude and perception of medical and dental undergraduates about antimicrobial stewardship. Indian J Pharmacol. 2015 Nov;47(6):676-9.

8. Kumar BVD, Kalpana L. A comparative study to assess the awareness of antibiotic resistance amongst 
first and second year medical undergraduate students in a medical college. Int $\mathbf{J}$ Basic Clin Pharmacol. 2018;7:1567-71.

9. Badar V, Parulekar VV, Garate P. Study of knowledge, attitude and practice amongst medical professionals about antimicrobial stewardship in tertiary care teaching hospital in India: a questionnaire based study. Int J Basic Clin Pharmacol. 2018;7:5117.

10. CDC. Core Elements of Hospital Antibiotic Stewardship Programs. Atlanta, GA: US Department of Health and Human Services, CDC; 2014. Available at: http://www/cdc.gov/getsmart/healthcare/inpatientstewardship. Accessed 25th September 2018.

11. Tayyab K, Shahzadi I, Mukhtar F, Shahid HA, Tahir I, Gohar H. Knowledge and Perceptions of Medical Students Regarding Antibiotic Use. PJMHS. 2017;11(1):145-9.

12. Yashin AN, Thakuria N,Narzary H, Satnami D, Paul N. A questionnaire based survey on the knowledge, attitude and practices about antimicrobial resistance and usage among the MBBS students and doctors of a tertiary care teaching Hospital in Silchar, Assam, India. Int J Basic Clin Pharmacol. 2018;7:1630-6.

13. Shubha R, Madhav K. Savkar, Manjunath GN. Self medications pattern among dentists with antibiotics. J Evolution Med Dental Sci. 2013;2(46):9037-41.
14. Shaik T, Meher BR. A questionnaire based study to assess the knowledge, attitude and practice (KAP) of rationale use of antibiotics among undergraduate dental students in a tertiary care dental hospital of South India. Int J Basic Clin Pharmacol. 2017;6:3125.

15. Hu Y, Wang X, Tucker JD, Little P, Moore M, Fukuda $\mathrm{K}$, et al. Knowledge, attitude, and practice with respect to antibiotic use among Chinese medical students: A multicentre cross-sectional study. Int J Environmental Res Public Health. 2018 Jun 4;15(6): 1165.

16. Abbo LM, Cosgrove SE, Pottinger PS, Pereyra M, Sinkowitz-Cochran R, Srinivasan A, et al. Medical Students' Perceptions and Knowledge About Antimicrobial Stewardship: How Are We Educating Our Future Prescribers? Clin Infectious Dis. 2013 Sep;57(5):631-8.

Cite this article as: Chundru J, Shailendra D, Tirumalareddy RR, Kumari H, Kovilakonda VP. Knowledge, perception and attitude of undergraduate and post-graduate medical students about antimicrobial use, resistance and stewardship at a tertiary care teaching hospital in rural Telangana, India. Int J Basic Clin Pharmacol 2019;8:193-9. 